\title{
Efecto terapéutico del alprostadil en pacientes con isquemia crítica terminal de los miembros inferiores ${ }^{\text {th }}$
}

\author{
Alfredo J. Karles-Ernotte ${ }^{a}$, Aníbal Bermúdez-Posada ${ }^{a}$, \\ Héctor M. Rincón-Sánchez ${ }^{a}$, Andrea T. Padilla-Castro ${ }^{a, b}$, Katherine Drews-Elger ${ }^{b, c}$, \\ Mauricio Novoa-Leal ${ }^{a}$ y Heinz G. Hiller-Correa ${ }^{\mathrm{a}, \mathrm{b}, *}$
}

\author{
a Corazón Instituto Especializado, Clínica El Rosario, Medellín, Colombia \\ ${ }^{\text {b } F a c u l t a d ~ d e ~ M e d i c i n a, ~ G r u p o ~ d e ~ I n v e s t i g a c i o ́ n ~ d e ~ E n f e r m e d a d e s ~ V a s c u l a r e s, ~ U n i v e r s i d a d ~ C E S, ~ M e d e l l i ́ n, ~ C o l o m b i a ~}$ \\ ' Facultad de Medicina, Grupo de Investigación Ciencias Básicas, Universidad CES, Medellín, Colombia
}

Recibido el 24 de noviembre de 2015; aceptado el 21 de abril de 2016

Disponible en Internet el 2 de junio de 2016

\author{
PALABRAS CLAVE \\ Enfermedad arterial \\ periférica; \\ Isquemia; \\ Prostaglandinas; \\ Reperfusión
}

\begin{abstract}
Resumen
Objetivo: El manejo de la isquemia crítica de los miembros inferiores representa un reto para el cirujano vascular debido a la alta tasa de amputaciones y mortalidad. Las opciones de manejo actuales: puente femorodistal, angioplastia con o sin la colocación de Stents y la resección de la placa con láser o de manera mecánica, presentan a largo plazo una tasa de éxito muy baja y un número de amputaciones supracondíleas que continúa siendo elevado.

Métodos: Para este estudio prospectivo se reclutaron 173 pacientes con diagnóstico de estadio avanzado, con isquemia crítica de miembro inferior quienes fueron tratados con alprostadil (60 - $120 \mathrm{mcgr} /$ día) por vía intravenosa sistémica por 28 días. La respuesta se midió clínicamente por mejoría del llenado capilar y con el uso de la escala análoga visual del dolor.

Resultados: Al momento del alta hospitalaria el 94.3\% de los pacientes mejoró el puntaje en la escala análoga visual del dolor $(\mathrm{p}<0.0001)$. El seguimiento a más de un año del tratamiento con alprostadil mostró que el $\mathbf{9 7 \%}$ de los pacientes mejoró significativamente su estadio de isquemia, evitándose así una amputación mayor. No se observó respuesta al tratamiento en pacientes previamente intervenidos por vía endovascular (5 pacientes).

Conclusiones: El tratamiento de pacientes con isquemia crítica de miembro inferior con alpostadil por infusión intravenosa, con bolos diarios de entre 60 y $120 \mathrm{mcg}$ durante 28 días, este medicamento es seguro y presenta mínimos efectos secundarios. Esta terapia mejora sustancialmente el estadio funcional de Rutherford en estos pacientes y evita amputaciones mayores. (c) 2016 Sociedad Colombiana de Cardiología y Cirugía Cardiovascular. Publicado por Elsevier España, S.L.U. Este es un artículo Open Access bajo la licencia CC BY-NC-ND (http:// creativecommons.org/licenses/by-nc-nd/4.0/).
\end{abstract}

\footnotetext{
Los autores declaran que no han recibido fuentes de apoyo externas para el desarrollo de la investigación.

* Autor para correspondencia.

Correo electrónico: heinzhiller@gmail.com (H.G. Hiller-Correa).
} 


\section{KEYWORDS}

Peripheral artery

disease;

Ischemia;

Prostaglandins;

Reperfusion

\section{Therapeutic effect of alprostadil in patients with critical lower limb ischemia}

\begin{abstract}
Objetive: Management of critical lower limb ischemia represents a challenge for the vascular surgeon due to the high rate of amputations and mortality. Current management options include femorodistal bypass, angioplasty with or without stent and laser or mechanical resection of the plaque. They present a low success rate in the long run and a number of supracondylar amputations that still remains high.

Methods: This prospective study included 173 patients diagnosed with advanced stage critical lower limb ischemia who were treated with systemic intravenous alprostadil (60 - $120 \mathrm{mcg} /$ day) during 8 days. Response was measured clinically with improvement of capillary refill and using the visual analog scale for pain.

Results: Upon discharge $94.3 \%$ of patients improved their visual analogue scale score for pain $(p<0.0001)$. Follow-up for more than a year of alprostadil treatment revealed that $97 \%$ of patients significantly improved their ischemia status, thus avoiding further amputation. No response to treatment was observed in patients who had previously undergone endovascular surgery (5 patients).

Conclusions: Treating patients with critical lower limb ischemia with intravenous alprostadil, administering daily doses of between 60 and 120 mcg during 28 days shows that this drug is safe and causes minimal secondary effects. This therapy significantly improves Rutherford's function state in these patients and avoids further amputations.

(c) 2016 Sociedad Colombiana de Cardiología y Cirugía Cardiovascular. Published by Elsevier España, S.L.U. This is an open access article under the CC BY-NC-ND license (http:// creativecommons.org/licenses/by-nc-nd/4.0/).
\end{abstract}

\section{Introducción}

El manejo de la isquemia crítica de los miembros inferiores representa un reto especial para el cirujano vascular. Se estima que cada año se presentan entre 500 y 1.000 casos por cada millón de personas en el mundo ${ }^{1}$, y de estos el 40-50\% de los pacientes serán amputados por encima de la rodilla con una mortalidad del $25-30 \%^{2,3}$.

La isquemia crítica, es el estado más avanzado de la enfermedad, se define como dolor en reposo continúo por más de 2 semanas, la ulceración por enfermedad arterial oclusiva crónica y la pérdida de una extremidad ${ }^{4}$. La causa de la isquemia se encuentra entre los factores de riesgo descritos para todas las enfermedades ateroscleróticas, como: fumar o estar expuesto al humo de la leña, la dislipidemia y la diabetes mellitus. El objetivo del tratamiento es mejorar el flujo arterial a la extremidad afectada para lograr un cierre de las úlceras, evitar la amputación mayor y mejorar la calidad de vida y sobrevida a largo plazo ${ }^{5}$. En la actualidad existen diversas opciones de manejo como: el puente femorodistal, la angioplastia con o sin la colocación de Stents y la resección de la placa con láser o de manera mecánica. No obstante, la tasa de éxito de estos métodos es muy baja a largo plazo y el número de amputaciones supracondíleas sigue siendo elevado ${ }^{6-8}$. En pacientes con claudicación intermitente, la extremidad ha tenido tiempo de desarrollar una circulación colateral efectiva, permitiendo perfundir la extremidad hasta el punto que muchos pacientes sin pulsos palpables pueden estar asintomáticos. Se ha propuesto como principal factor asociado el tiempo en el cual se desarrolla la isquemia, permite al cuerpo compensar la ausencia de flujo sanguíneo reclutando vasos sanguíneos colaterales. Esta circulación colateral, se podría en teoría acceder para mejorar la perfusión en una situación crítica9

El beneficio del uso de las prostaglandinas en pacientes con dolor crónico en ausencia de isquemia crítica se ha demostrado en varios estudios que incluyeron más de 7 estudios aleatorizados comparativos con un total de 643 pacientes $^{10,11}$. En especial los trabajos de Creutzig et al., publicado en el año 2004, demostraron que es seguro aplicar esta opción de tratamiento en pacientes con isquemia crítica severa $^{12}$. Sin embargo, no hay estudios recientes del uso de las prostaglandinas, específicamente el alprostadil en pacientes con isquemia crítica severa, es decir, el paciente con dolor agudo que no mejora con el reposo y tiene por ende un alto riesgo de amputación.

En los trabajos publicados por Mourikis et al., se sigue un protocolo en el cual el fármaco se aplica por vía intraarterial en infusión lenta ${ }^{13}$. Las ventajas de este método radican en la vasodilatación y el reclutamiento de circulación colateral de manera inmediata con mejoría de la isquemia. No obstante, en ocasiones es necesario el uso de la anestesia raquídea, pues la vasodilatación directa en la arteria causa intenso dolor (comunicación personal). Los resultados de estos estudios son prometedores, ya que la tasa de amputación reportada es menor al $20 \%{ }^{13}$.

En este trabajo reportamos la evaluación de una serie prospectiva desde el año 2011, con pacientes en estado avanzado de la enfermedad, aplicando el alprostadil por vía intravenosa sistémica como última opción terapéutica previo a la amputación mayor de una extremidad. El efecto a largo plazo es muy similar a la aplicación intraarterial como lo describe Creutzig en su metaanálisis ${ }^{12}$, sin embargo, el efecto vasodilatador completo con la infusión intravenosa 
es solo observable luego de 2-3 días de la infusión. La mayor ventaja de este protocolo radica en la fácil aplicación, infusión indolora y ausencia de efectos cardiovasculares importantes observados durante la misma. Esto permite que en países donde exista un adecuado servicio de salud en casa, esta terapia puede ser aplicada de manera ambulatoria en el hogar.

En este trabajo reportamos los resultados del seguimiento a un año de los pacientes en estado avanzado de isquemia crítica del miembro inferior de cualquier causa (diabetes mellitus, aterosclerosis, tromboangeítis obliterante, escleroderma) tratados con alprostadil por vía intravenosa sistémica en la Clínica El Rosario en Medellín, Colombia.

\section{Materiales y métodos}

El trabajo en curso es un estudio prospectivo de una serie de casos que se inició en mayo de 2011. Se reclutaron pacientes con los siguientes criterios de inclusión: pacientes mayores de 18 años, con enfermedad arterial estadio Rutherford 4-6, que no son candidatos a cirugía reconstructiva o tratamiento endovascular, pacientes con: hipertensión arterial, falla renal en cualquier estadio, diabetes mellitus y pacientes con cirugía o intervención arterial previa. Se excluyeron pacientes con: falla cardiaca congestiva, fracción de eyección menor al 30\%, alteración de la conciencia y cáncer en cualquier estadio.

\section{Recolección de variables}

A todos los pacientes se les realizó: la ecocardiografía, el duplex arterial y la arteriografía de aorta en los miembros inferiores (en pacientes sin falla renal) para dar cumplimiento con los criterios de inclusión y exclusión. Se recolectaron todas las variables al ingreso del paciente a la clínica, mediante anamnesis realizada por el médico de urgencias. Se interconsultaron con medicina interna aquellos pacientes con comorbilidades para el manejo simultáneo de las mismas. Todos los pacientes fueron evaluados por el cirujano vascular de la Clínica El Rosario.

\section{Tratamientos adicionales}

Los pacientes con ulceraciones fueron desbridados quirúrgicamente y en casos de infección se inició la antibioticoterapia de acuerdo al protocolo institucional. Se definió la amputación mayor como la necesidad de realizar esta cirugía por encima del tobillo y proximal a este (infra o supracondílea).

\section{Terapia con alprostadil}

Desde el ingreso del paciente, su diagnóstico y la inclusión en el estudio (en promedio 10 horas), se inició el tratamiento con el alprostadil con $60 \mathrm{mcg} /$ día como dosis estándar de inicio, en infusión continúa durante 3 horas en solución salina normal por la vena periférica. La dosis se incrementó 20 mcg cada 2 días en caso de no observarse respuesta satisfactoria, hasta máximo $120 \mathrm{mcg} /$ día.
Todos los pacientes fueron hospitalizados por un período mínimo de 3 días, el medicamento se aplicó durante 28 días continuos y se les realizó seguimiento durante un año.

\section{Variables}

Los datos demográficos recolectados incluyeron: la edad, el género, las comorbilidades, las intervenciones y las amputaciones mayores previas. Entre el ingreso y la salida del paciente, se evaluó diariamente clínicamente: el llenado capilar, el estadio de la enfermedad y se hizo la evaluación del dolor mediante el uso de la escala análoga del dolor. Posterior a la intervención y previo al alta se realizó una nueva medición del dolor, se registró: el tiempo de hospitalización, las complicaciones asociadas a la terapia y el número de amputaciones mayores. De acuerdo con estas variables se definió el tiempo de respuesta al medicamento.

\section{Análisis estadístico}

Se hizo un análisis descriptivo de cada una de las variables. Los resultados se presentan como el promedio y desviación estándar. Se realizó una comparación del efecto del tratamiento sobre el dolor de los pacientes mediante la prueba de $t$ de Student pareada. La base de datos fue recolectada en una hoja de Excel encriptada y se aplicó el análisis estadístico utilizando el programa EPIDAT ver 4.1.

\section{Consideraciones éticas}

Todos los pacientes firmaron consentimiento informado previo a la utilización del medicamento. Se les hizo énfasis en la naturaleza de la enfermedad, la cual en otras circunstancias llevaría a la amputación de la extremidad. El protocolo cumple con las guías de ética de la declaración de Helsinki de 1975, reflejado en la aprobación por parte del Comité Institucional de la Clínica El Rosario de Medellín, Colombia.

\section{Resultados}

Hasta la fecha de publicación de este artículo se reportan 173 pacientes que completaron el año de seguimiento (hombres $n=122$ ) de un total de 187 pacientes que actualmente se han incluido en la serie. El promedio de edad fue de 71,4 años (máx. 81, mín. $22 \mathrm{SD} \pm 1,3$ ). 62 pacientes (36\%) se presentaron al ingreso con ulceración por necrosis isquémica. La distribución por estadio de severidad de la isquemia Rutherford fue la siguiente: estadio $462 \%$, estadio $533 \%$, estadio $63 \%$. En el grupo de los pacientes en estadio 4 se encontraron $2 \%(n=3)$ de pacientes con tromboangeítis obliterante. La comorbilidad más frecuente fue la hipertensión arterial, seguido de la diabetes mellitus tipo 2 (tabla 1). El 17\% de los pacientes estaban anticoagulados por fibrilación auricular y tromboembolismo pulmonar previo. La dosis de alprostadil máxima aplicada por paciente fue de $120 \mathrm{mcg} /$ día.

El valor de la escala visual análoga al inicio del tratamiento fue de un promedio de 9,4 (mín. 8,7, máx. 10 SD \pm $0,075)$. El tiempo de respuesta fue en promedio de 3,9 días (mín. 2, máx. $6 \mathrm{SD} \pm 0,166)$ de los cuales un 12\% $(\mathrm{n}=21)$ requirió titulación de la dosis. Al alta, el valor promedio de 
Tabla 1 Características de los pacientes al entrar en el estudio

\begin{tabular}{|c|c|}
\hline Característica & \% Pacientes (n) \\
\hline Edad & $71,44(\operatorname{Max} 81, \min 22)$ \\
\hline Hombres & $70(n=122)$ \\
\hline Rutherford 4 & $62(n=108)$ \\
\hline Rutherford 5 & $33(n=57)$ \\
\hline Rutherford 6 & $3(n=5)$ \\
\hline $\begin{array}{l}\text { Enfermedad de Buerger } \\
\text { (Tromboangeitis }\end{array}$ & $2(n=3)$ \\
\hline Obliterante) & $88,4(n=153)$ \\
\hline Hipertensión Arterial & $15(n=26)$ \\
\hline Enfermedad Coronaria & $2,3(n=4)$ \\
\hline Diabetes Mellitus & $35,8(n=62)$ \\
\hline Infección ${ }^{a}$ & $32,9(n=57)$ \\
\hline Amputación Previa ${ }^{\mathrm{b}}$ & $2,9(n=5)$ \\
\hline Tratamiento Endovascular Previo ${ }^{c}$ & $5,8(n=10)$ \\
\hline Cirugía Previa ${ }^{d}$ & $2,3(n=4)$ \\
\hline \multicolumn{2}{|c|}{$\begin{array}{l}\text { a Infección de la extremidad afectada o infección de la } \\
\text { ulceración. } \\
\text { b Amputación infra o supracondílea previa, } \\
\text { c Angioplastia y/o colocación del stent en arterias de miembros } \\
\text { inferiores. } \\
\text { d Puente fémoro-poplíteo o fémoro-tibial previo, }\end{array}$} \\
\hline
\end{tabular}

\section{Escala dolor inicio}

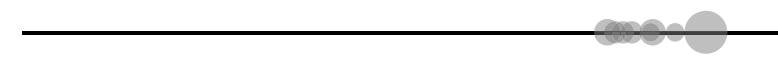

\section{Escala dolor seguimiento}

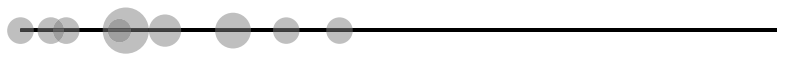

\section{$\left|\begin{array}{llll|l|l|l|l|l|l|l|}1 & \mid 2 & 3 & 4 & 5 & 6 & \mid 7 & 8 & \mid 9 & 10\end{array}\right|$}

Figura 1 Escala análoga de dolor al inicio del tratamiento y al momento del seguimiento al alta. El tamaño de los círculos es proporcional al volumen de pacientes en ese punto de la escala.

la escala análoga visual fue de 2,8 (mín. 1, máx. 5,2 SD \pm $0,18)$, lo cual representó una mejoría significativa del dolor $(p<0,0001)$ (fig. 1). En la mayoría de los casos solo se requirió un desbridamiento y en un $13 \%$ fue necesario realizar una amputación menor (definida como amputación de un dígito).

El tratamiento falló en 5 pacientes (3\%) los cuales requirieron amputación mayor (amputación infra o supracondílea) (fig. 2). A pesar de aumentar las dosis del alprostadil no se logró controlar la isquemia, y la enfermedad progresó hasta la isquemia irreversible de toda la extremidad. Al verificar los antecedentes de estos pacientes a todos se les había realizado una angioplastia infracondílea en los meses previos al ingreso. En un paciente se tuvo que suspender el protocolo del alprostadil por presentar edema agudo del pulmón a las 24 horas del inicio de la infusión, y subsecuentemente requirió una amputación supracondílea. Se revisó el ecocardiograma de ingreso y reportaba una fracción de eyección del $38 \%$. No se observaron otros eventos adversos a la administración del medicamento.

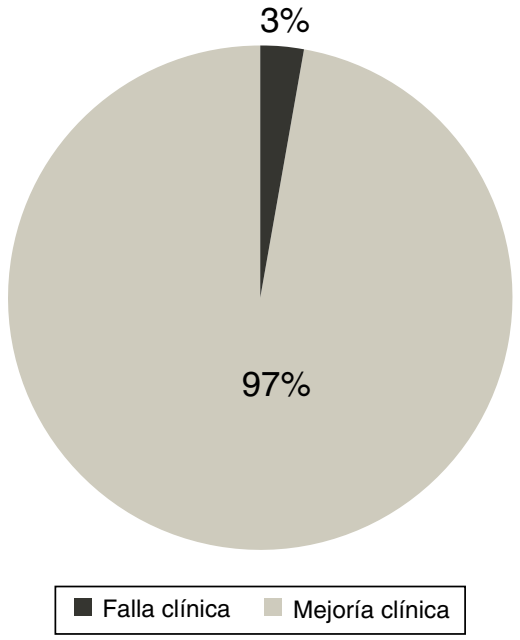

Figura 2 Respuesta al tratamiento con alprostadil.

El tiempo promedio de epitelialización de las úlceras fue de 26 días (mín. 15 días, máx. 68 días).

La tasa de amputación mayor (como se definió previamente) fue del $3 \%$ en el período de seguimiento, es decir, $3 \%$ en un año. Los pacientes que no requirieron amputación mejoraron la escala de isquemia de Rutherford de la siguiente manera: Un $67 \%$ mejoró a un estadio 2, $22 \%$ estadio 3 y un $1 \%$ quedaron en un estadio 4 , con dolor de reposo intermitente el cual mejoró en los meses subsecuentes con la adición del cilostazol 100 mg por vía oral cada 12 horas.

\section{Análisis de subgrupos}

En el grupo de estudio se incluyeron pacientes con otras patologías (no aterosclerótica) como causa de isquemia de las extremidades. Se trataron 5 pacientes con tromboangeítis obliterante en los cuales las arteriografías no mostraron flujo por debajo de las rodillas; estos pacientes recibieron 60 mcg del alprostadil por vía venosa por día durante 28 días. Los 5 pacientes fueron hospitalizados durante 3 días, al cabo del los cuales se continuó el manejo con un programa de salud en casa hasta completar los 28 días del protocolo. Todos los pacientes presentaron mejoría significativa del dolor luego de la primera dosis y al cabo de dos días pudieron deambular en el piso de la hospitalización. Todos los pacientes de este subgrupo mejoraron su deambulación hasta el punto de no tener claudicación intermitente tras un año de seguimiento. Tampoco se registraron amputaciones en este grupo.

Se incluyeron 3 pacientes con enfermedad de Raynaud y con escleroderma que causó pérdida parcial de los dígitos de las manos en hospitalizaciones previas y que ingresaron por agudización de su patología. A estos pacientes se les administró el alprostadil 60 mcg intravenoso durante 28 días con el mismo esquema propuesto. Al cabo de 4 días de infusión se observó mejoría del dolor, y detención del proceso de necrosis de los dígitos. En el seguimiento durante los meses posteriores, se observó una adecuada cicatrización de los dígitos de las manos. Después de un año de seguimiento no se presentaron más episodios de isquemia en las manos afectadas, ni en otras localizaciones. 


\section{Discusión}

El uso de las prostaglandinas en los pacientes con isquemia ha sido controversial, ya que hay una falta de estudios de impacto, con amplias muestras y aleatorizados que demuestren un verdadero beneficio. Esta controversia se debe en parte al uso de estos análogos de la prostaglandina en los pacientes con claudicación intermitente, en los cuales no se demostró un beneficio claro ${ }^{14}$.

En el año 2004 se publicaron estudios que mostraron un efecto benéfico de las prostaglandinas en la circulación afectada por la aterosclerosis. Entre estos trabajos se destaca el estudio de Morishita et al., que demostró el aumento del flujo arterial en pacientes con aterosclerosis de las extremidades luego de la aplicación de la prostaglandina ${ }^{15}$. El estudio de Petronella et al., comparó los efectos del tratamiento médico con las prostaglandinas vs. simpatectomía lumbar, método que se ha utilizado con frecuencia en los pacientes con dolor refractario ${ }^{16}$. Su grupo demostró la superioridad del manejo médico en controlar el dolor por isquemia en estos pacientes. Además, se observó mejoría de la clase funcional de estos pacientes aunque el objetivo principal del estudio no fuera ese. Los efectos de las prostaglandinas en la circulación fueron ampliamente descritos por Hohlfeld et al. ${ }^{17}$. Según su trabajo, las prostaglandinas no solo tienen un efecto vasodilatador, sino también actúan por medio de la regulación génica, al inhibir la expresión de las moléculas de adhesión pro inflamatorias como la ICAM-1 y VCAM-1, TNF alpha, MCP-1 y los factores de crecimiento CYR61 y CTGF. Esto explicaría los posibles efectos a largo plazo del tratamiento con las prostaglandinas-E1. Existen otros reportes que no encontraron beneficio con el tratamiento con las prostaglandinas como el publicado por Llorente et al., en el año 2008 que no demostró mejoría en 18 pacientes de los 24 estudiados $^{18}$. Es posible que en este último no se hayan utilizado las dosis adecuadas para mejorar la perfusión, tampoco se escaló en las dosis de acuerdo a la respuesta obtenida y, además, se incluyeron un número muy pequeño de pacientes para sacar conclusiones significativas. Cochrane publicó similares resultados en una revisión sobre el uso de las prostaglandinas en la isquemia crítica el cual determinó que si hay un efecto positivo pero que faltan estudios de mayor magnitud para determinar la efectividad $^{19}$.

En el presente estudio, se reclutaron 173 pacientes que estaban en estadios avanzados de la enfermedad, no se incluyeron pacientes claudicantes. El seguimiento a más de un año mostró que la gran mayoría (97\%) mejoró su estadio de isquemia y evitó una amputación mayor. El protocolo que utilizamos se basó en los trabajos de Milcan et al. ${ }^{20}$, en el cual se demuestra que el uso de las prostaglandinas protege a los tejidos de una lesión por reperfusión, lo cual fue una de las preocupaciones iniciales. Ninguno de los pacientes presentó deterioro de la función renal durante el estudio. Al contrario, en muchos pacientes se observó una mejoría en el valor de la creatinina, lo cual puede explicarse por la mejoría de la isquemia muscular lo que evita su degradación. La medición de la escala análoga visual se correlaciona adecuadamente con el progreso clínico del paciente y el cuestionario de impedimento de deambulación descrito por Matsuo et al. ${ }^{21}$. Este hallazgo nos permitió validar este método como una forma costo-efectiva de determinar el efecto del tratamiento con el alprostadil.

En los pacientes con falla terapéutica se encontró como factor común que todos habían sido intervenidos previamente con angioplastia de las arterias comprometidas. Es posible, que las intervenciones en los lechos afectados causaran una disfunción endotelial no reversible como está descrito en el trabajo publicado por Chaabane et al. ${ }^{22}$. Un resultado no esperado del estudio fue la mejoría clínica de los pacientes con tromboangeítis obliterante. No solo se logró evitar la amputación que habitualmente acompaña a esta patología, sino que también se logró una mejoría significativa de su estadio de isquemia, hasta el punto que los pacientes solo claudicaban a más de 500 metros y no tenían dolor en reposo. Igual efecto se observó en los pacientes con escleroderma y enfermedad de Raynaud lo cual genera la inquietud de si se deberían utilizar las prostaglandinas en estos pacientes como tratamiento inicial. Estos resultados son comparables a los obtenidos por Salera et al., el cual estudió el cambio de flujo en pacientes con escleroderma luego de una infusión de prostavasin ${ }^{23}$ y mostró que el aumento de flujo a nivel de la arteria braquial, vena porta y arteria esplénica fueron significativos.

El presente estudio muestra que el uso del alprostadil no solo genera vasodilatación inicial con reclutamiento de circulación colateral, sino también una acción antiinflamatoria que tiene efectos a largo plazo. El seguimiento a más de un año de los pacientes que tuvieron una respuesta favorable inicial, mostró que ninguno de ellos deterioró su estado funcional y que ninguno sufrió una amputación mayor al cabo de este tiempo. En 13 pacientes fue necesario repetir la dosis de alprostadil a los 6 meses por recurrencia de claudicación a menos de 50 metros, pero no se presentó reaparición de ulceraciones o dolor en reposo.

La serie presentada en este estudio es una de las más grandes en la literatura y continúa creciendo, ya que el centro vascular donde se realiza se ha convertido en centro de referencia para el manejo de esta patología dado los resultados favorables que se han obtenido.

\section{Conclusiones}

El uso del alprostadil en infusión intravenosa con bolos diarios de entre 60 y $120 \mathrm{mcg}$ durante 28 días es seguro, con mínimos efectos secundarios. Hay un claro beneficio para los pacientes con isquemia crítica al mejorar el estadio funcional de Rutherford lo cual les permite mejorar su calidad de vida, y evitar amputación en un $97 \%$ de los casos utilizando el protocolo propuesto. Los pacientes que no tuvieron respuesta al tratamiento fueron aquellos que habían sido intervenidos previamente por vía endovascular. Continuamos evaluando el costo beneficio de esta terapia a largo plazo comparado con la intervención quirúrgica y endovascular para la isquemia crítica de los miembros inferiores.

\section{Responsabilidades éticas}

Protección de personas y animales. Los autores declaran que los procedimientos seguidos se conformaron a las normas éticas del comité de experimentación humana 
responsable y de acuerdo con la Asociación Médica Mundial y la Declaración de Helsinki.

Confidencialidad de los datos. Los autores declaran que han seguido los protocolos de su centro de trabajo sobre la publicación de datos de pacientes.

Derecho a la privacidad y consentimiento informado. Los autores han obtenido el consentimiento informado de los pacientes y/o sujetos referidos en el artículo. Este documento obra en poder del autor de correspondencia.

\section{Conflicto de intereses}

Todos los autores declaran que no existen conflictos de intereses.

\section{Reconocimientos y agradecimientos}

Los autores desean agradecer a la Clínica El Rosario por el apoyo en la hospitalización y seguimiento de los pacientes. Adicionalmente, desean agradecer a las diferentes especialidades de apoyo de la clínica, que permitieron un manejo integral de todos los pacientes en este trabajo.

\section{Bibliografía}

1. Mangiafico RA, Mangiafico M. Medical treatment of critical limb ischemia: current state and future directions. Current vascular pharmacology. 2011;9:658-76.

2. Lumsden AB, Davies MG, Peden EK. Medical and endovascular management of critical limb ischemia. Journal of endovascular therapy: an official journal of the International Society of Endovascular Specialists. 2009;16:1131-62, http://dx.doi.org/10.1583/08-2657

3. Egorova NN, Guillerme S, Gelijns A, Morrissey N, Dayal $\mathrm{R}$, McKinsey JF, et al. An analysis of the outcomes of a decade of experience with lower extremity revascularization including limb salvage, lengths of stay, and safety. Journal of vascular surgery. 2010;51:878-85, http://dx.doi.org/10.1016/j.j vs. 2009;10.102, 885 e871.

4. Novo S, Coppola G, Milio G. Critical limb ischemia: definition and natural history. Current drug targets. Cardiovascular \& haematological disorders. 2004;4:219-25.

5. Slovut DP, Sullivan TM. Critical limb ischemia: medical and surgical management. Vascular medicine. 2008;13:281-91, http: / /dx.doi.org/10.1177/1358863X08091485

6. Conte MS. Understanding objective performance goals for critical limb ischemia trials. Seminars in vascular surgery. 2010;23:129-37, http://dx.doi.org/10.1053/ j.semvascsurg.2010.06.001

7. Diamantopoulos A, Katsanos K. Treating femoropopliteal disease: established and emerging technologies. Seminars in interventional radiology. 2014;31:345-52, http://dx.doi.org/10.1055/s-0034-1393971

8. Schamp KB, Meerwaldt R, Reijnen MM, Geelkerken RH, Zeebregts CJ. The ongoing battle between infrapopliteal angioplasty and bypass surgery for critical limb ischemia. Annals of vascular surgery. 2012;26:1145-53, http://dx.doi.org/10.1016/j.avsg.2012.02.006
9. Brodmann M. The angiosome concept in clinical practice: implications for patient-specific recanalization procedures. The Journal of cardiovascular surgery. 2013;54:567-71.

10. Bandiera G, Forletta M, Di Paola FM, Cirielli C. PGE(1) short term therapy in critical lower limb ischemia. International angiology: a journal of the International Union of Angiology. 2003;22:58-63.

11. Mlekusch W, Schillinger $M$, Sabeti S, Al-Awami $M$, Gschwandtner M, Minar E. Effects of intravenous prostaglandin E1 on arterial compliance: a randomized controlled trial. VASA. Zeitschrift fur Gefasskrankheiten. 2004;33:131-6, http://dx.doi.org/10.1024/0301-1526.33.3.131

12. Creutzig A, Lehmacher W, Elze M. Meta-analysis of randomised controlled prostaglandin E1 studies in peripheral arterial occlusive disease stages III and IV. VASA. Zeitschrift fur Gefasskrankheiten. 2004;33:137-44, http://dx.doi.org/10.1024/0301-1526.33.3.137

13. Chatziioannou A, Dalakidis A, Katsenis K, Koutoulidis V, Mourikis D. Intra-arterial prostaglandin e(1) infusion in patients with rest pain: short-term results. The Scientific World Journal. 2012:803678, http://dx.doi.org/10.1100/2012/803678

14. Robertson L, Andras A. Prostanoids for intermittent claudication. The Cochrane database of systematic reviews. 2013;4:CD000986, http://dx.doi.org/10.1002/ 14651858.CD000986.pub3

15. Makino H, Aoki M, Hashiya N, Yamasaki K, Hiraoka K, Shimizu H, et al. Increase in peripheral blood flow by intravenous administration of prostaglandin E1 in patients with peripheral arterial disease, accompanied by up-regulation of hepatocyte growth factor. Hypertension research: official journal of the Japanese Society of Hypertension. 2004;27:85-91.

16. Petronella P, Freda F, Nunziata L, Antropoli M, Manganiello A, Cutolo PP, et al. Prostaglandin E1 versus lumbar sympathectomy in the treatment of peripheral arterial occlusive disease: randomised study of 86 patients. Nutrition, metabolism, and cardiovascular diseases: NMCD. 2004;14:186-92.

17. Schror K, Hohlfeld T. Mechanisms of anti-ischemic action of prostaglandin E1 in peripheral arterial occlusive disease. VASA. Zeitschrift fur Gefasskrankheiten. 2004;33:119-24.

18. Llorente Gutiérrez J, González Fernández MA, Fuentes Irigoyen R, Jiménez Caballero E. [Intravenous PGE-1 in peripheral vascular obstructive disease: use over a 6-month period]. Farmacia hospitalaria: órgano oficial de expresión científica de la Sociedad Española de Farmacia Hospitalaria. 2004;28:188-91.

19. Ruffolo AJ, Romano M, Ciapponi A. Prostanoids for critical limb ischaemia. The Cochrane database of systematic reviews. 2010:CD006544, http://dx.doi.org/10.1002/ 14651858.CD006544.pub2

20. Milcan A, Arslan E, Bagdatoglu OT, Bagdatoglu C, Polat G, Kanik $A$, et al. The effect of alprostadil on ischemia-reperfusion injury of peripheral nerve in rats. Pharmacological research. 2004; 49:67-72.

21. Matsuo H, Shigematsu H. Patient-based outcomes using the Walking Impairment Questionnaire for patients with peripheral arterial occlusive disease treated with Lipo-PGE1. Circulation journal: official journal of the Japanese Circulation Society. 2010;74:365-70.

22. Chaabane C, Otsuka F, Virmani R, Bochaton-Piallat ML. Biological responses in stented arteries. Cardiovascular research. 2013;99:353-63, http://dx.doi.org/10.1093/cvr/cvt115

23. Salera D, Argalia G, Giuseppetti GM. Hemodynamic effects of a prostacyclin analog (Prostavasin) in systemic scleroderma patients. La Radiología Médica. 2005;110:106-14. 